\title{
Erratum to: Visual priming through a boost of the target signal: Evidence from saccadic landing positions
}

\author{
M. Meeter · S. Van der Stigchel
}

Published online: 17 January 2014

(C) Psychonomic Society, Inc. 2014

\section{Erratum to: Atten Percept Psychophys \\ DOI 10.3758/s13414-013-0516-z}

The publisher regrets having omitted the abstract and key words in the original article. The abstract and key words appear below.

\begin{abstract}
Searching for a target is slower when target features change from trial to trial than when they are repeated. Although heavily studied, it is still not wholly clear what process is influenced by such visual priming. Here, we introduce a new measure to study priming. When a target and distractor are in close proximity, fast saccades generally fall in between the two, a finding known as the global effect. We elicited global effect saccades to study the effects of repeating target or distractor colors on overt attention. Saccades landed closer to a target or distractor in the color of a previous target, suggesting that priming enhances target color signals. This was true even for the fastest eye movements, in the range of express saccades. Distractor color repetition, on the other hand, had no effect, at least in isolation. Visual priming is, we conclude, at least partly the result of boosting perceptual target signals.
\end{abstract}

Keywords Attention · Priming · Eye movements · Visual attention

The online version of the original article can be found at $\mathrm{http}: / / \mathrm{dx}$.doi.org/ 10.3758/s13414-013-0516-z.

M. Meeter $(\bowtie)$

Department of Cognitive Psychology, VU University Amsterdam,

V.d. Boechorststraat 1, 1081 BT Amsterdam, The Netherlands

e-mail:m.meeter@vu.nl

S. Van der Stigchel

Experimental Psychology, Helmholtz Institute, Utrecht University,

Utrecht, The Netherlands 